\title{
DINÂMICA E PRODUÇÃO DE UMA FLORESTA SOB REGIME DE MANEJO SUSTENTÁVEL NA AMAZÔNIA CENTRAL
}

\author{
Marcos Antônio Silva de Souza ${ }^{1}$, Celso Paulo de Azevedo², Cíntia Rodrigues de Souza ${ }^{2}$, \\ Mabiane França ${ }^{3}$, Elias Lourenço Vasconcelos Neto ${ }^{4}$ \\ ${ }^{1}$ Universidade Federal do Amazonas, Programa de Pós-Graduação em Ciências Florestas e Ambientais, Manaus, Amazonas, Brasil - \\ mass.florestal@gmail.com \\ ${ }^{2}$ Embrapa Amazônia Ocidental, Pesquisa e Desenvolvimento, Manaus, Amazonas, Brasil - celso.azevedo@embrapa.br \\ /cintia.souza@embrapa.br \\ Faculdade Metropolitana de Manaus, Manaus, Amazonas, Brasil - mabiane@ hotmail.com \\ ${ }^{4}$ Universidade Nilton Lins, Manaus, Amazonas, Brasil - netoelvn@gmail.com \\ Recebido para publicação: 29/09/2015 - Aceito para publicação: 17/02/2017
}

\begin{abstract}
Resumo
Avaliou-se os efeitos de curto e médio prazos do sistema silvicultural policíclico, na dinâmica e produção, em uma floresta ombrófila densa no estado do Amazonas, tendo como base três Unidades de Produção Anual da área de manejo florestal da empresa Mil Madeiras Preciosas em Itacoatiara, AM, exploradas entre os anos de 1996 a 1998, com intensidade de exploração média de 20,02 $\mathrm{m}^{3} \cdot \mathrm{ha}^{-1}$. Os inventários florestais contínuos foram realizados antes e após a exploração nos anos de 1996, 1997, 1998, 2001 e 2014 em 41 parcelas permanentes de 1 hectare, onde foram mensuradas todas as árvores com DAP igual ou superior a $15 \mathrm{~cm}$. Registrou-se a presença de 195 espécies, sendo 55 comerciais. Em valores médios, antes da exploração, foram registradas 297,65 árvores.ha ${ }^{-1}$, equivalente a área basal de $26,42 \mathrm{~m}^{2} \cdot \mathrm{ha}^{-1} \mathrm{e}$ o volume de $393,62 \mathrm{~m}^{3} \cdot \mathrm{ha}^{-1}$. A floresta apresentou taxa média de ingresso de $3,20 \%$. ano ${ }^{-1}$, assim como $1,47 \%$.ano ${ }^{-1}$ de mortalidade. Considerando apenas as espécies de valor comercial, as taxas foram de 2,50\%.ano ${ }^{-1}$ e $1,30 \%$.ano ${ }^{-1}$ para ingresso e mortalidade, respectivamente. $\mathrm{O}$ incremento em volume foi de $4,63 \mathrm{~m}^{3} \cdot \mathrm{ha}^{-1} \cdot \mathrm{ano}^{-1}$, dos quais $1,69 \mathrm{~m}^{3} \cdot \mathrm{ha}^{-1} \cdot \mathrm{ano}^{-1}$ de espécies comerciais. A floresta estudada apresentou estoques em área basal e volume do povoamento total e das espécies comerciais, equivalentes aos registrados antes da exploração, indicando que a intensidade de corte aplicada está favorecendo a recuperação do estoque madeireiro para um segundo ciclo de corte.
\end{abstract}

Palavras-chave: Manejo florestal; incremento; dinâmica; parcelas permanentes.

\begin{abstract}
Dynamic and production of a forest under sustainable management in central amazon. Evaluated the short effects and medium term of polycyclic silvicultural system, dynamics and production, in a dense rain forest in the state of Amazonas, based on three Annual Production Units (APU) of the forest management area of the company Mil Madeiras Preciosas in Itacoatiara, AM, explored between the years 1996 to 1998, with an average exploitation intensity of $20,02 \mathrm{~m}^{3} \cdot \mathrm{ha}^{-1}$. The continuous forest inventories were done before and after logging in 1996, 1997, 1998, 2001 and 2014 in 41 permanent plots of 1 hectare where all trees with DAP equal to or greater than $15 \mathrm{~cm}$ were measured. The presence of 195 species was recorded, being 55 commercial. On average values, before the logging, 297.65 trees.ha $^{-1}$ were recorded, equivalent to basal area of $26.42 \mathrm{~m}^{2} \cdot \mathrm{ha}^{-1}$ and the volume of $393.62 \mathrm{~m}^{3} \cdot \mathrm{ha}^{-1}$. The forest showed average rate of $3.20 \%$.year ${ }^{-1}$, and $1.47 \%$.year ${ }^{-1}$ mortality. Considering only the species of commercial value, the rates were $2.50 \%$ year $^{-1}$ and $1.30 \%$ year $^{-1}$ for admission and mortality, respectively. The increase in volume was $4.63 \mathrm{~m}^{3} \cdot \mathrm{ha}^{-1}$. $\mathrm{year}^{-1}$, of which $1.69 \mathrm{~m}^{3} \cdot$ ha $^{-1}$.year ${ }^{-1}$ commercial species. The forest studied had stocks in basal area and volume of the total population and commercial species, equivalent to those recorded before the logging, indicating that the applied shear intensity is favoring the recovery of the timber stock into a second cutting cycle.

Keywords: Forest management; increment; dynamic; permanent plots.
\end{abstract}

\section{INTRODUÇÃO}

O Brasil abriga a maior extensão de floresta tropical continua do mundo (FAO, 2010) e para as regiões com extensa cobertura florestal, o manejo sustentável é apontado como uma das melhores opções de crescimento econômico sem degradação ambiental. Entretanto, para que seja alcançada a sustentabilidade da produção madeireira são necessários planejamentos eficazes, que garantam fornecimento de madeira de forma contínua, além de benefícios econômicos, sociais e ambientais.

O sistema de manejo policíclico é o que melhor se adaptou à estrutura da floresta Amazônica, promovendo uma boa regeneração, apesar do ponto negativo deste método ser a exploração seletiva das espécies,

FLORESTA, Curitiba, PR, v. 47, n. 1, p. 55 - 63, jan. / mar. 2017.

Souza, M. A. S. de et al.

ISSN eletrônico 1982-4688

DOI: $10.5380 /$ rf.v47i1.43312 
onde são exploradas apenas as de valor no mercado, provocando pressão desigual nas entre as populações dessas espécies e as demais (SOUZA, 2012). A fim de manter a sustentabilidade desse sistema, De Graaf (1986) sugere ampliar a lista de espécies a serem exploradas e extrair somente os indivíduos maduros, além da aplicação de tratamentos silviculturais, que induzam a regeneração e o maior crescimento das espécies comercialmente valiosas, reduzindo a pressão seletiva nas populações das mesmas.

Deste modo torna-se imprescindível o conhecimento dos efeitos das operações florestais na dinâmica das florestas manejadas, principalmente em termos de biomassa e composição das espécies. Toledo et al. (2011), cita que a dinâmica das florestas após o manejo é difícil de ser prevista em função da alta heterogeneidade (solo, topografia, estrutura da floresta original, variações climáticas e biodiversidade). Logo, um dos pontos importantes que devem ser investigados nas florestas manejadas é a taxa de crescimento das árvores, combinada à identificação e quantificação dos fatores que influem sobre este crescimento.

As análises do crescimento, ingresso e mortalidade, quando tratadas em conjunto, são imprescindíveis para o entendimento dos processos de evolução do ecossistema florestal (FURTADO, 2009), sendo estas de fundamental importância para o planejamento da produção e determinação dos ciclos de corte de florestas tropicais, pois as taxas de incremento positivo, combinadas a frequentes ingressos, compõem um dos indicativos de que a floresta possui características favoráveis ao manejo para produção madeireira (OLIVEIRA, 2005).

O método usual de obtenção de resultados consistentes de dinâmica de florestas, é por meio de estudos de longo prazo, realizados em parcelas permanentes (SILVA et al., 1996). Mesmo que as parcelas permanentes sejam consideradas onerosas, de difícil instalação e medição, o estudo de dinâmica de florestas vem sendo feito seguindo esta metodologia a décadas e, de acordo com as pesquisas realizadas até então, continua sendo a melhor forma de obter respostas confiáveis sobre o desenvolvimento dessas florestas a longo prazo (LIMA, 2010).

Neste trabalho, buscou-se avaliar os efeitos de curto e médio prazos do sistema silvicultural policíclico, na dinâmica e produção, em uma floresta ombrófila densa no Estado do Amazonas, tendo como base três Unidades de Produção Anual da área de manejo florestal da empresa Mil Madeiras Preciosas, exploradas entre os anos de 1996 a 1998.

\section{MATERIAL E MÉTODOS}

\section{Localização}

Este estudo foi desenvolvido a partir de dados de 41 parcelas permanentes localizadas na Fazenda Dois Mil, pertencente à área de Manejo Florestal Sustentável da Empresa Mil Madeiras Preciosas, localizada entre os municípios de Itacoatiara, Silves e Itapiranga, no Amazonas, nas coordenadas geográficas de $02^{\circ} 30^{\prime} \mathrm{S}$ e $59^{\circ} 00^{\prime}$ $\mathrm{W}$ e $03^{\circ} 00^{\prime} \mathrm{S}$ e $58^{\circ} 30^{\prime} \mathrm{W}$ Gr. Hoje a área de manejo florestal da empresa é de 202.104,76 hectares, explorada por meio do sistema policíclico CELOS adaptado no Brasil, com um ciclo de corte definido pela legislação para 35 anos (PMFS/Mil Madeiras, 2013).

O clima da região, baseado na classificação de Köppen-Geiger (Am), é tropical chuvoso monçônico, com altas precipitações durante quase todo o ano, diferindo apenas por apresentar maiores quedas pluviométricas durante três meses do ano (KOTTEK et al., 2006). A vegetação é classificada como Floresta Ombrófila Densa, caracterizada por indivíduos arbóreos de grade porte, com lianas lenhosas e epífitas (IBGE, 2012). Os solos da região estão classificados no grupo dos Latossolos Amarelos Distróficos, de acordo com o Mapa Geral de Solos do Brasil (RADAMBRASIL, 1978).

\section{Descrição das Parcelas Permanentes}

As parcelas permanentes foram instaladas com a dimensão de $100 \mathrm{~m}$ x $100 \mathrm{~m}$ (1 ha). Cada parcela foi dividida em 100 subparcelas de $10 \mathrm{~m}$ x $10 \mathrm{~m}$. Nestas, de cada árvore com diâmetro a 1,30 m do solo (DAP) $\geq$ $15 \mathrm{~cm}$, foram avaliados os seguintes parâmetros: 1) identificação botânica; 2) DAP; 3) classe de identificação do fuste (CIF); 4) situação silvicultural; 5) presença de danos/defeitos; 6) grau de iluminação da copa; 7) forma da copa; 8) presença de cipós; 9) classe de floresta e 10) coordenadas cartesianas (SILVA et al., 2005).

As 41 parcelas permanentes utilizadas no estudo estão divididas entre as UPAs B, C e D, exploradas nos anos de 1996, 1997 e 1998 respectivamente, com intensidade média de 20,02 $\mathrm{m}^{3} \cdot \mathrm{ha}^{-1}$ nos indivíduos de DAP $\geq$ $50 \mathrm{~cm}$. As parcelas floram medidas um ano antes da exploração e remedidas em 1998 na UPA B, assim como em 2001 e 2014 nas três UPAs.

\section{Análise dos dados}

Com base nas medições e remedições, foram determinados os incrementos médios em diâmetro, área basal e volume por hectare, bem como as taxas de mortalidade e de ingresso.

As taxas de ingresso e mortalidade foram obtidas por meio das seguintes equações: 


$$
I(\%)=\frac{i}{a a} \cdot 100 \quad M(\%)=\frac{m}{a a} \cdot 100
$$

em que: I a taxa de ingresso (\%), i o número de árvores que ingressaram, aa o número de árvores da medição anterior, $\mathrm{M}$ a taxa de mortalidade (\%) e m o número de árvores que morreram.

Os incrementos periódicos anuais IPA $\mathrm{DAP}_{\text {, IPA }}$ e IPA $\mathrm{I}_{\mathrm{V}}$, referentes ao DAP, área basal e volume, respectivamente, foram calculados por meio das seguintes equações:

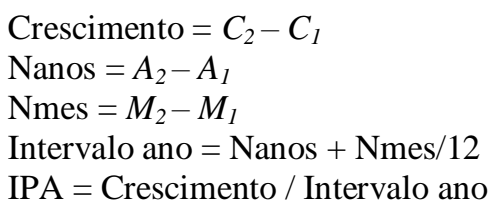

em que: $\boldsymbol{C}_{2}$ e $\boldsymbol{C}_{\boldsymbol{l}}$ mensurações no final do período e no início do período, respectivamente, da variável de interesse, $\boldsymbol{A}_{2}$ e $\boldsymbol{A}_{\boldsymbol{l}}$ os anos no final e no início do período, respectivamente, $\boldsymbol{M}_{2}$ e $\boldsymbol{M}_{\boldsymbol{l}}$ os meses no final e no início do período, respectivamente, IPA o incremento periódico anual.

A área basal foi obtida por meio das seguintes fórmulas:

$$
G=\sum_{i=1}^{n} g_{i} \quad g_{i}=\frac{\pi \cdot D A P^{2}}{40.000}
$$

em que: $\boldsymbol{G}$ a área basal (m²/ha), $\boldsymbol{g}_{i}$ a área da seção transversal individual, $\boldsymbol{\pi}$ pi (constante matemática) e o $\boldsymbol{D A} \boldsymbol{P}$ (diâmetro a 1,30 $\mathrm{m}$ do solo em $\mathrm{cm}$ ).

O volume individual (vi) foi calculado através da equação ajustada por Lima (2010), feita para a região central de Manaus:

$$
v i=0,001176 *(D A P)^{1,99868}\left(R^{2}=0,89 \text { e incertez } a=4,0 \%\right)
$$

em que: vi o volume comercial com casca $\left(\mathrm{m}^{3}\right)$ e $\boldsymbol{D A P}$ o diâmetro à altura do peito $(\mathrm{cm})$.

Realizou-se o teste t de Student a 95\% de probabilidade, comparando as variáveis volume, área basal, diâmetro e número de espécies antes e após a exploração.

$$
\begin{array}{ccc}
d=x_{2}-x_{1} & {[1]} & \bar{d}=\frac{\sum d}{n} \\
S^{2}=\frac{\sum d^{2}-\frac{\left(\sum d\right)^{2}}{n}}{n-1} & {[3]} & t=\frac{\bar{d}}{\sqrt{\frac{S^{2}}{n}}}
\end{array}
$$

em que: $\boldsymbol{d}$ a diferença das unidades (N, DAP, G e V) entre as duas medições, $\boldsymbol{x}_{\boldsymbol{1}}$ o valor das unidades (N, DAP, G e V) antes da exploração, $\boldsymbol{x}_{2} \mathrm{o}$ valor das unidades (N, DAP, G e V) depois da exploração, $\boldsymbol{t}$ o valor do teste $\mathrm{t}$ de Student e $\boldsymbol{n}$ o Grau de liberdade.

Também foram realizadas análises de correlação simples, utilizando o coeficiente de correlação linear de Pearson, e análise de variância (ANOVA) a 95\% de probabilidade, com os fatores definidos pelas variáveis categóricas iluminação da copa, forma da copa e CIF, com dados não balanceados, para Incremento periódico

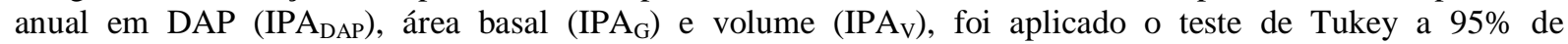
probabilidade para comparação das médias.

\section{RESULTADOS E DISCUSSÃO}

\section{Número de Árvores, Área Basal e Volume}

Considerando a médias das três UPAs estudadas (DAP $\geq 15 \mathrm{~cm}$ ), antes da exploração, foram registradas 297,65 árvores.ha ${ }^{-1}( \pm 31,24)$, equivalente a área basal de $26,42 \mathrm{~m}^{2} \cdot \mathrm{ha}^{-1}( \pm 2,96)$ e o volume de $393,62 \mathrm{~m}^{3} \cdot \mathrm{ha}^{-1}( \pm$ 
44,06), sendo 157,92 $\mathrm{m}^{3} \cdot \mathrm{ha}^{-1}( \pm 33,18)$ de espécies comerciais. Em 2001, 2 e 4 anos após a exploração, a área basal passou a ser de $22,54 \mathrm{~m}^{2} \cdot \mathrm{ha}^{-1}( \pm 2,66)$, representando uma redução de $3,89 \mathrm{~m}^{2}$.ha $\mathrm{ha}^{-1}$, considerando as árvores extraídas e a mortalidade decorrente da exploração. O motivo maior dessa redução foi a exploração realizada na área e a mortalidade de árvores decorrente das atividades de derruba e arraste das toras. Oliveira e Braz (2006), observaram uma redução de 2,51 m².ha $\mathrm{ma}^{-1}, 2$ anos após a exploração na Amazônia Ocidental.

Em 2014, 16 e 18 anos após a exploração, todas as UPAs apresentaram aumento significativo nas três variáveis, onde a média do volume passou a ser de $366,10 \mathrm{~m}^{3} \cdot \mathrm{ha}^{-1}( \pm 33,79)$ e $134,75 \mathrm{~m}^{3} \cdot \mathrm{ha}^{-1}( \pm 28,38)$ para as espécies comerciais, sendo estatisticamente igual aos valores observados antes da exploração.

A distribuição do volume médio entre as classes diamétricas, em todo o período de acompanhamento, considerando as três UPAs, pode ser observada na figura 1.

Volume - Todas as Espécies

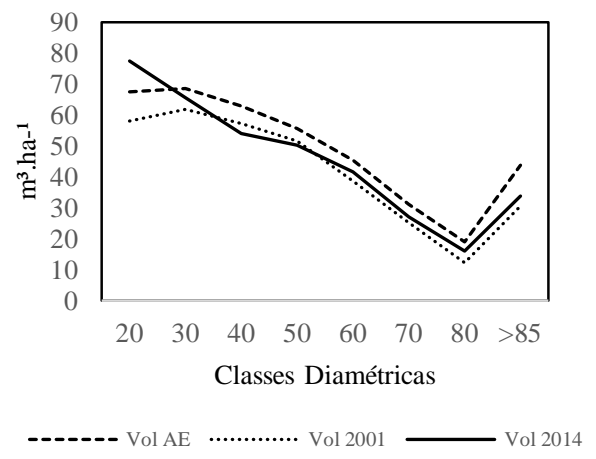

Volume - Espécies Comerciais

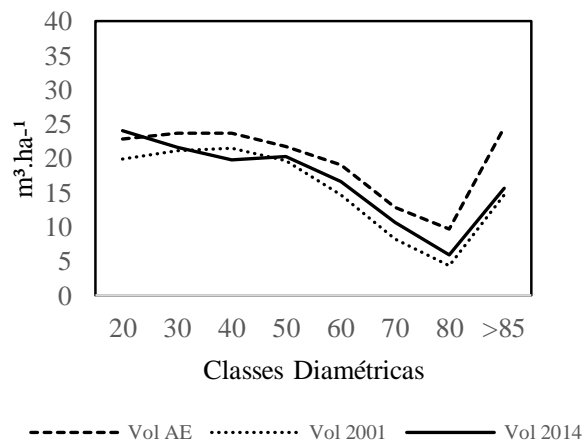

Figura 1. Média do volume total e das espécies comerciais, entre as classes diamétricas, registrada antes da exploração (Vol AE), em 2001 e em 2014 (16 a 18 anos após a exploração), nos 41 hectares amostrados da área de Manejo Florestal da empresa Mil Madeiras, Itacoatiara, AM.

Figure 1. Average of the total volume of commercial species and among the diameter classes, recorded before operation (Vol AE) in 2001 and in 2014 (16 to 18 years after logging) in 41 hectares sampled the Forest Management Area the company Mil Madeiras Preciosas, Itacoatiara, AM.

Comparando o período entre o primeiro levantamento (antes da exploração) e o último, realizado em 2014 (16 e 18 depois), observou-se alterações na dinâmica entre as classes. A recuperação do volume ocorreu de forma mais efetiva nas primeiras classes $(15-35 \mathrm{~cm})$. Esse fato era esperado, uma vez que essas classes não foram alvos da extração de madeira, embora tenham sido reduzidas em consequência dos danos provocados pela exploração (Figura 1). Nas classes acima de $45 \mathrm{~cm}$ de diâmetro, o aumento do volume observado ainda não foi suficiente para alcançar os valores presentes antes da extração das árvores, entretanto, percebe-se a tendência de retorno aos níveis encontrados na área antes da exploração, principalmente considerando o volume das espécies comercias entre 55 e $75 \mathrm{~cm}$ de diâmetro.

\section{Taxa de ingresso e mortalidade}

O balanço do ingresso e mortalidade de todas as espécies e das espécies comerciais, considerando a média dos três compartimentos (41 hectares amostrados), está representado na figura 2.

Nota-se que a taxa de mortalidade, excluindo-se as árvores extraídas, só foi superior à de ingressos no período logo após a exploração, tanto para todas as espécies, quanto para espécies de valor comercial (Figura 2). Essa taxa elevada deve-se, além da mortalidade natural, à ocasionada pelo processo de extração das árvores comerciais, que acarretou danos as remanescentes, principalmente às árvores de menor porte $(\mathrm{DAP} \leq 30 \mathrm{~cm})$ devido às atividades de derruba, guinchamento e arraste das toras. Oliveira (2005) e Lopes (1993) comentam que a alta taxa de mortalidade de árvores, logo após a exploração, é causada principalmente pela derruba e pelos danos resultantes da operação de arraste.

No segundo período o balanço passou a ser positivo, ou seja, o número de ingressos superou a mortalidade, onde as taxas de 3,2\%.ano ${ }^{-1}$ e 2,5\%. ano $^{-1}$ para as espécies comerciais (Figura 2) foram semelhantes às encontradas por Higuchi et al. (1997) na região de Manaus (2,9\%.ano ${ }^{-1}$ a 4,2\%.ano ${ }^{-1}$ ) e por Silva et al. (1995), 11 anos após a exploração, na Floresta Nacional do Tapajós $\left(3,1 \%\right.$.ano $\left.{ }^{-1}\right)$. 
Todas As Espécies

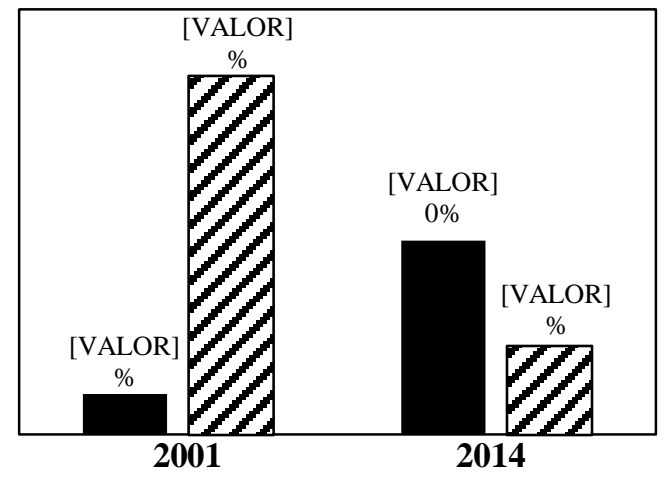

- Ingresso $\square$ Mortalidade

\section{Espécies Comerciais}

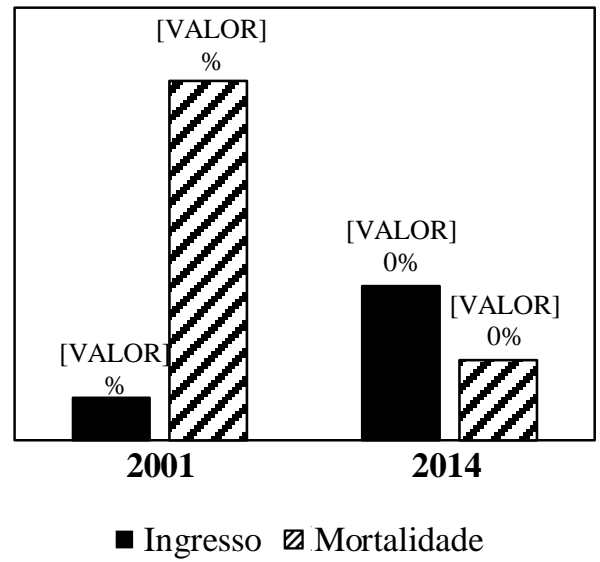

Figura 2. Balanço entre o ingresso e mortalidade total e das espécies comerciais, com valores registrados entre 2 e 4 anos após a exploração (2001), comparados ao período de 16 e 18 anos após a exploração (2014), nos 41 hectares amostrados de uma Floresta sob regime de Manejo Florestal da empresa Mil Madeiras, Itacoatiara, AM.

Figure 2. Balance between the inflow and total mortality and commercial species, with amounts recorded between 2-4 years after the operation (2001), compared to the period16 to 18 years after logging (2014) in 41 hectares sampled a forest under management system of the company Mil Madeiras, Itacoatiara, AM.

O balanço positivo observado em 2014 comprova que o aumento da mortalidade devido à exploração, está sendo compensado pelo aumento do número de ingressos, principalmente entre as espécies de valor comercial, o que valoriza a floresta em termos de potencial madeireiro para o próximo ciclo de corte.

\section{Incremento periódico anual -IPA em diâmetro, área basal e volume}

Os valores do incremento periódico anual nas UPAs B, C e D em termos de DAP, área basal e volume, para todas as espécies e para o grupo de espécies comerciais, após as atividades de exploração madeireira, são apresentados na tabela 1.

As taxas médias de incremento registradas em volume foram de $4,63 \mathrm{~m}^{3} \cdot \mathrm{ha}^{-1} \cdot \mathrm{ano}^{-1}$ para todas as espécies e de 1,69 $\mathrm{m}^{3} \cdot \mathrm{ha}^{-1}$.ano ${ }^{-1}$ para as espécies comerciais. Estes valores foram semelhantes às de $4,67 \mathrm{~m}^{3}$.ha ${ }^{1}$.ano ${ }^{-1}$ observada por Nascimento (2012), 21 anos após a exploração, e de Oliveira e Braz (2006), que observaram um incremento de $1,06 \mathrm{~m}^{3} \cdot \mathrm{ha}^{-1} \cdot \mathrm{ano}^{-1}$ para as espécies comerciais. Ambos estudos foram realizados em florestas manejadas na Amazônia Ocidental.

Em florestas manejadas experimentalmente, Teixeira et al. (2007) observaram incremento de $5,60 \mathrm{~m}^{3} \cdot \mathrm{ha}^{-1} \cdot$ ano $^{-1}$ para todas as espécies e Higuchi et al. (1997) $1 \mathrm{~m}^{3} \cdot \mathrm{ha}^{-1} \cdot$ ano $^{-1}$ para as espécies comerciais com $\mathrm{DAP} \geq 50 \mathrm{~cm}$, ambos estudos na região de Manaus, Estado do Amazonas. Oliveira (2005) observou incremento de $1,9 \mathrm{~m}^{3} \cdot \mathrm{ha}^{-1} \cdot$ ano $^{-1}$ também para as espécies comerciais, num tratamento definido como exploração de leve intensidade (DAP $\geq 55 \mathrm{~cm}$ e desbaste de 20\%), na Amazônia Oriental.

As taxas de incremento registradas em DAP foram de $0,27 \mathrm{~cm}^{-a n o} \mathrm{o}^{-1}$ para todas as espécies e $0,30 \mathrm{~cm} \cdot \mathrm{ano}^{-1}$ para as de valor comercial. Estas taxas foram inferiores às de $0,36 \mathrm{~cm}$.ano ${ }^{-1}$ encontrada por Carvalho et al. (2004), na FLONA do Tapajós, 8 anos após a exploração e a de $0,5 \mathrm{~cm}$.ano $\mathrm{o}^{-1}$ encontrada por Silva (2004), em exploração de impacto reduzido, na região de Paragominas, PA. Oliveira (2005) também observou uma taxa superior $\left(0,34 \mathrm{~cm} \cdot\right.$ ano $\left.^{-1}\right)$ para as espécies comerciais, entretanto, em tratamentos com exploração seguida de desbastes, na Amazônia Oriental.

Para melhor observar a influência da exploração madeireira nas taxas de crescimento das árvores em diferentes tamanhos, os valores de incremento foram plotados em um gráfico por classe de diâmetro (Figura 3).

$\mathrm{Na}$ figura 3, é possível observar que a média do incremento tende a aumentar à medida que aumenta a classe de diâmetro, alcançando o ponto máximo nas árvores de 75 e $85 \mathrm{~cm}$, com os maiores incrementos registrados $\operatorname{logo}$ após a exploração. Essa mesma tendência foi observada por Cunha (2009), sugerindo que as árvores de maior diâmetro, que têm mais acesso à luz, são as mais beneficiadas com a abertura do dossel decorrente da exploração. 
Tabela 1. Incremento periódico anual (IPA) em DAP (média das árvores), Área basal e Volume, das árvores com diâmetro $\geq 15 \mathrm{~cm}$, entre as UPAs B, C e D, na área amostral de 41 hectares da área de Manejo Florestal da empresa Mil Madeiras, Itacoatiara, AM.

Table 1. Increase annual periodical (IPA) in DAP (trees' average), basal area and volume, of trees with diameter $\geq 15 \mathrm{~cm}$, between APUs B, C and D in the sample area of 41 hectares of Forest Management Area of the company Mil Madeiras, Itacoatiara, AM.

\begin{tabular}{|c|c|c|c|c|c|c|c|}
\hline \multirow[b]{2}{*}{ UPA } & \multirow[b]{2}{*}{ Período } & \multicolumn{3}{|c|}{ Todas as Espécies } & \multicolumn{3}{|c|}{ Espécies Comerciais } \\
\hline & & 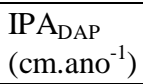 & $\begin{array}{l}\text { IPA Área Basal } \\
\left(\mathrm{m}^{2} \cdot \mathrm{ha}^{-1} \cdot \mathrm{ano}^{-1}\right)\end{array}$ & $\begin{array}{l}\text { IPA Volume } \\
\left(\mathrm{m}^{3} \cdot \mathrm{ha}^{-1} \cdot \text { ano }^{-1}\right)\end{array}$ & 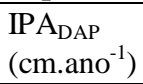 & $\begin{array}{l}\text { IPA Área Basal } \\
\left(\mathrm{m}^{2} \cdot \mathrm{ha}^{-1} \cdot \mathrm{ano}^{-1}\right)\end{array}$ & $\begin{array}{l}\text { IPA Volume } \\
\left(\mathrm{m}^{3} \cdot \mathrm{ha}^{-1} \cdot \text { ano }^{-1}\right)\end{array}$ \\
\hline \multirow{3}{*}{ B } & 1996-1998 & 0,2727 & 0,3595 & 5,3536 & 0,2714 & 0,1213 & 1,8067 \\
\hline & $1998-2001$ & 0,2591 & 0,2976 & 4,4317 & 0,2725 & 0,1049 & 1,5620 \\
\hline & $2001-2014$ & 0,2850 & 0,3227 & 4,8058 & 0,2898 & 0,1199 & 1,7849 \\
\hline \multirow{2}{*}{ C } & $1997-2001$ & 0,1931 & 0,2392 & 3,5639 & 0,3835 & 0,0944 & 1,4062 \\
\hline & 2001-2014 & 0,2592 & 0,2911 & 4,335 & 0,2810 & 0,1191 & 1,7742 \\
\hline \multirow{2}{*}{ D } & $1998-2001$ & 0,2981 & 0,3468 & 5,1645 & 0,3036 & 0,1170 & 1,7425 \\
\hline & 2001-2014 & 0,3045 & 0,3206 & 4,7746 & 0,3192 & 0,1182 & 1,7603 \\
\hline Média & & 0,2673 & 0,3110 & 4,6327 & 0,3029 & 0,1135 & 1,6909 \\
\hline
\end{tabular}

Todas As Espécies

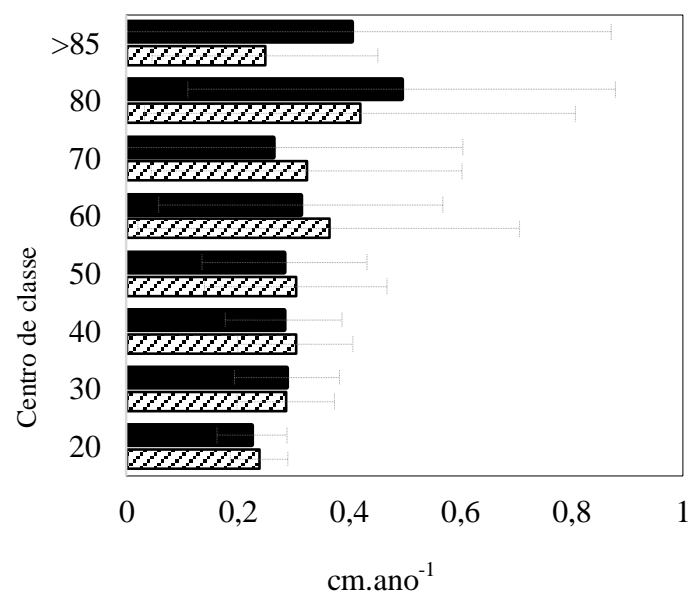

IPA - AE $\square$ IPA- 2001 a 2014

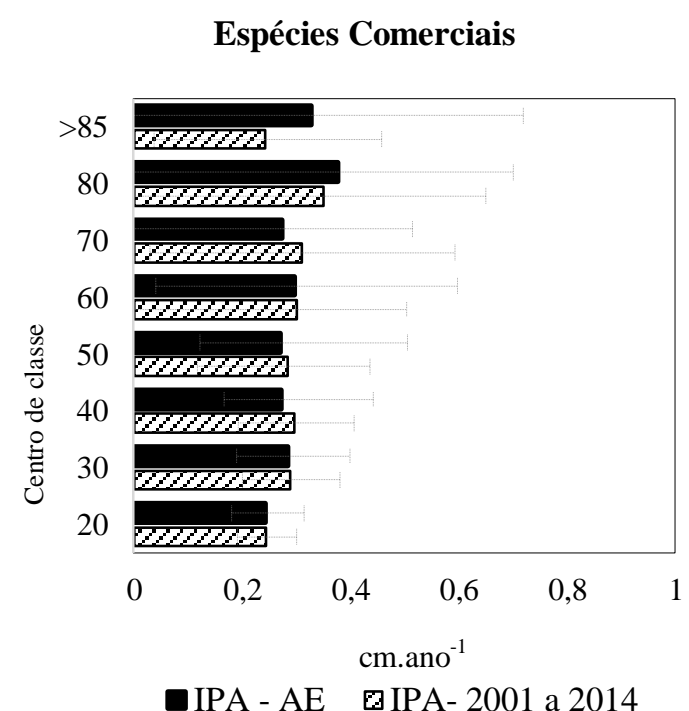

IPA - AE $\square$ IPA- 2001 a 2014

Figura 3. Incremento periódico anual - $\mathrm{IPA}_{\mathrm{DAP}}$ de todas as espécies e das espécies comerciais, entre as classes diamétricas, no período 1996-2001 (AE) e no período 2001-2014 (16 e 18 anos após a exploração), nos 41 hectares amostrados de uma Floresta Ombrófila Densa sob regime de Manejo Florestal da empresa Mil Madeiras, Itacoatiara- AM. As barras representam o desvio padrão.

Figure 3. Increase annual periodic -IPA $A_{D A P}$ of all species and commercial species, among diameter classes, period from 1996 to 2001 (AE) and period from 2001 to 2014 (16 and 18 years after logging) in 41 hectares sampled from a dense rain forest under forest management system of the company Mil Madeiras, Itacoatiara- AM. The bars represent the standard deviation.

Observa-se ainda na figura 3 o aumento do desvio padrão do incremento médio entre as árvores de maior diâmetro, indicando maior exposição desses indivíduos às variantes ambientais que influenciam o crescimento, tais como, posição sociológica, presença de cipó, danos, entre outros.

Analisando as variáveis com potencial em influir sobre o crescimento (Tabela 2), observou-se que a relação do crescimento foi altamente significativa $(p>0,0001)$ com o grau de iluminação da copa e a forma da copa, significativa ao nível de $95 \%$ de probabilidade com a classe de identificação do fuste (CIF) e não

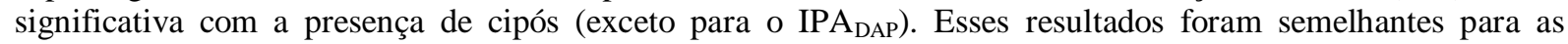
observações tomadas em 2001 (2 e 4 anos após a exploração) e em 2014, 16 a 18 anos após a exploração. 
Tabela 2. Matriz de correlação de Pearson das variáveis categóricas registradas em 2001 e 2014, em relação ao Incremento Periódico Anual (IPA), nos 41 hectares amostrados de uma Floresta Ombrófila Densa pertencente a área de Manejo Florestal da empresa Mil Madeiras, Itacoatiara, AM.

Table 2. Pearson's correlation matrix of categorical variables recorded in 2001 and 2014 in relation to Increment Annual Periodic Review (IPA), in 41 hectares sampled from a forest of Management Area of company Mil Madeiras, Itacoatiara, AM.

\begin{tabular}{|c|c|c|c|c|}
\hline \multicolumn{5}{|c|}{ OBSERVAÇÕES EM 2001} \\
\hline & Iluminação de Copa & Formada Copa & Cipó & $* \mathrm{CIF}$ \\
\hline \multirow{3}{*}{$I P A-_{D A P}$} & $\mathrm{r}=-0,104$ & $\mathrm{r}=-0,067$ & $r=-0,023$ & $\mathrm{r}=-0,024$ \\
\hline & $p<0,0001$ & $p<0,0001$ & $p=0,0162$ & $p=0,0122$ \\
\hline & $n=10113$ & $n=10113$ & $n=10113$ & $n=10113$ \\
\hline \multirow{3}{*}{$I P A-_{G}$} & $r=-0,228$ & $\mathrm{r}=-0,052$ & $r=0,004$ & $r=-0,020$ \\
\hline & $p<0,0001$ & $p<0,0001$ & $p=0,6576$ & $p=0,0439$ \\
\hline & $n=10113$ & $n=10113$ & $n=10113$ & $n=10113$ \\
\hline \multirow{3}{*}{$I P A-{ }_{\text {Vol }}$} & $\mathrm{r}=-0,227$ & $\mathrm{r}=-0,052$ & $\mathrm{r}=0,004$ & $\mathrm{r}=-0,020$ \\
\hline & $p<0,0001$ & $p<0,0001$ & $p=0,6594$ & $p=0,0438$ \\
\hline & $n=10113$ & $n=10113$ & $n=10113$ & $n=10113$ \\
\hline \multicolumn{5}{|c|}{ OBSERVAÇÕES EM 2014} \\
\hline & Iluminação de Copa & Formada Copa & Cipó & $* \mathrm{CIF}$ \\
\hline \multirow{3}{*}{$I P A-_{D A P}$} & $r=-0,1843$ & $r=-0,1397$ & $r=-0,0469$ & $r=-0,0274$ \\
\hline & $p<0,0001$ & $p<0,0001$ & $p<0,0001$ & $p=0,0120$ \\
\hline & $n=8344$ & $n=8344$ & $n=8344$ & $n=8344$ \\
\hline \multirow{3}{*}{$I P A-_{G}$} & $r=-0,2537$ & $r=-0,1111$ & $r=-0,0053$ & $r=-0,0214$ \\
\hline & $p<0,0001$ & $p<0,0001$ & $p=0,6220$ & $p=0,0497$ \\
\hline & $n=8344$ & $n=8344$ & $n=8344$ & $n=8344$ \\
\hline \multirow{3}{*}{$I P A-{ }_{V o l}$} & $r=-0,2537$ & $r=-0,1111$ & $r=-0,0054$ & $r=-0,0214$ \\
\hline & $p<0,0001$ & $p<0,0001$ & $p=0,6193$ & $p=0,0495$ \\
\hline & $n=8344$ & $n=8344$ & $n=8344$ & $n=8344$ \\
\hline
\end{tabular}

*Classe e identificação do fuste

Com base nestes resultados, foi conduzida a análise de variância do crescimento com a forma e o grau de iluminação da copa e com o CIF (classe e identificação do fuste), entretanto, o CIF não apresentou diferença significativa ( $p=0,2349)$. Os resultados são apresentados nas tabelas 3 e 4.

Como esperado, a taxa média de crescimento foi maior entre os indivíduos com a copa completamente exposta à luz solar, ocorrendo um decréscimo à medida que esta exposição tornou-se parcial ou imperceptível. A mesma tendência foi observada por Vidal et al. (2002) na Amazônia Oriental e Cunha (2009) na Amazônia Ocidental. Esses autores citam que a luz solar, descrita pela posição sociológica, é um dos fatores decisivos que proporcionam maiores taxas de crescimento das árvores.

Tabela 3. Variação do IPA em DAP, Área Basal e Volume, em função da Iluminação de Copa, antes e após a exploração. Floresta sob manejo da empresa Mil Madeiras, Itacoatiara-AM. Os valores seguidos da mesma letra não diferem entre si pelo teste de Tukey ao nível de $95 \%$ de probabilidade.

Table 3. Variation of the IPA in DAP, Basal Area and Volume, depending on crown lighting before and after logging. Forest under management of company Mil Madeiras, Itacoatiara-AM. The values followed by the same letter do not differ by Tukey test at $95 \%$ probability.

\begin{tabular}{|c|c|c|c|c|}
\hline \multicolumn{5}{|c|}{ MEDIÇÃO EM 2001} \\
\hline Fator & $\mathbf{N}$ & IPA $A_{D A P}$ & $\mathbf{I P A}_{\mathbf{G}}$ & IPA $_{\text {Vol }}$ \\
\hline 1 Copa completamente exposta a luz & 1305 & $0,2903 \mathrm{a}$ & $0,0022 \mathrm{a}$ & $0,0328 \mathrm{a}$ \\
\hline 2 Copa parcialmente iluminada & 5775 & 0,2767 a & $0,0015 \mathrm{~b}$ & $0,0229 \mathrm{~b}$ \\
\hline 3 Copa completamente coberta & 3366 & $0,2205 \mathrm{~b}$ & $0,0008 \mathrm{c}$ & $0,0126 \mathrm{c}$ \\
\hline 4 Sem avaliação (árvore sem copa) & 30 & $0,1248 \mathrm{~b}$ & $0,0004 \mathrm{c}$ & $0,0065 \quad \mathrm{c}$ \\
\hline \multicolumn{5}{|c|}{ MEDIÇÃO EM 2014} \\
\hline Fator & $N$ & IPA ${ }_{\text {DAP }}$ & IPA $_{\mathbf{G}}$ & IPA $_{\text {Vol }}$ \\
\hline 1 Copa completamente exposta a luz & 1527 & $0,3929 \mathrm{a}$ & $0,0030 \mathrm{a}$ & $0,0446 \mathrm{a}$ \\
\hline 2 Copa parcialmente iluminada & 4559 & $0,2882 \mathrm{~b}$ & $0,0015 \mathrm{~b}$ & $0,0218 \mathrm{~b}$ \\
\hline 3 Copa completamente coberta & 2232 & $0,1825 \mathrm{c}$ & $0,0007 \mathrm{c}$ & $0,0103 \mathrm{c}$ \\
\hline 4 Sem avaliação (árvore sem copa) & 26 & $0,1353 \mathrm{c}$ & $0,0006 \mathrm{c}$ & $0,0087 \mathrm{c}$ \\
\hline
\end{tabular}

FLORESTA, Curitiba, PR, v. 47, n. 1, p. 55 - 63, jan. / mar. 2017.

Souza, M. A. S. de et al.

ISSN eletrônico 1982-4688

DOI: $10.5380 /$ rf.v47i1.43312 
Nas medições realizadas em 2001, 12,5\% das árvores amostradas (1305 indivíduos) encontravam-se com a copa recebendo luz solar total. Este percentual aumentou para 18,3\% (1527 indivíduos) em 2014. Isto indica que 16 e 18 anos após exploração o manejo continua proporcionando luz solar em maior quantidade para as árvores remanescentes, consequentemente favorecendo o crescimento e recuperação da volumetria original.

Em relação a forma da copa (Tabela 4) o maior incremento ocorreu nas árvores com a copa completa e bem distribuída (63,2\% dos indivíduos). Isso demonstra que a forma da copa exerce significativa influência na produção das árvores.

No segundo período estudado, com as medições realizadas em 2014, o percentual de árvores com a copa bem distribuída diminuiu ligeiramente para 58,8\% e o de árvores com a copa irregular passou de $33,1 \%$ para $36,0 \%$. Este fato demonstra estabilidade da estrutura física da floresta, em relação a área foliar dos indivíduos, comparada às observações realizadas logo após a exploração.

Tabela 4. Variação do IPA em DAP, Área Basal e Volume, em função da Forma da Copa, antes da exploração. Os valores seguidos da mesma letra não diferem entre si pelo teste de Tukey ao nível de $95 \%$ de probabilidade. Área amostral da área de Manejo Florestal da empresa Mil Madeiras, Itacoatiara, AM.

Table 4. Variation IPA in DAP, Basal Area and Volume, depending on the shape of the crown before the operation. The values followed by the same letter do not differ by Tukey test at $95 \%$ probability. Sample area of Forest Management Area company Mil Madeiras, Itacoatiara, AM.

\begin{tabular}{|c|c|c|c|c|}
\hline \multicolumn{5}{|c|}{ MEDIÇÃO EM 2001} \\
\hline Fator & $\mathbf{N}$ & IPA ${ }_{\text {DAP }}$ & IPA $_{G}$ & IPA $_{\text {Vol }}$ \\
\hline 1 Copa completa normal & 8386 & $0,2537 \mathrm{a}$ & $0,0013 \mathrm{a}$ & $0,0186 \mathrm{a}$ \\
\hline 2 Copa completa irregular & 1631 & $0,2187 \mathrm{~b}$ & $0,0011 \quad b$ & $0,0157 \quad b$ \\
\hline 5 Sem copa & 15 & 0,1179 bc & $0,0005 \mathrm{c}$ & $0,0080 \mathrm{bc}$ \\
\hline 3 Copa incompleta & 31 & $0,1144 \mathrm{bc}$ & $0,0004 \mathrm{c}$ & $0,0064 \mathrm{c}$ \\
\hline 4 Rebrotação & 52 & $0,0959 \mathrm{c}$ & $0,0004 \mathrm{c}$ & $0,0063 \mathrm{c}$ \\
\hline \multicolumn{5}{|c|}{ MEDIÇÃO EM 2014} \\
\hline Fator & $\mathbf{N}$ & IPA $A_{\text {DAP }}$ & $\mathbf{I P A}_{\mathbf{G}}$ & IPA $_{\text {Vol }}$ \\
\hline 1 Copa completa normal & 5021 & $0,3179 \mathrm{a}$ & $0,0018 \mathrm{a}$ & $0,0270 \mathrm{a}$ \\
\hline 2 Copa completa irregular & 2886 & $0,2309 \mathrm{~b}$ & $0,0012 \mathrm{~b}$ & $0,0174 \mathrm{~b}$ \\
\hline 5 Sem copa & 221 & 0,1720 bc & 0,0009 bc & 0,0131 bc \\
\hline 3 Copa incompleta & 170 & $0,1702 \mathrm{c}$ & $0,0008 \mathrm{bc}$ & $0,0116 \mathrm{bc}$ \\
\hline 4 Rebrotação & 46 & $0,1006 \mathrm{c}$ & $0,0005 \mathrm{c}$ & $0,0071 \mathrm{c}$ \\
\hline
\end{tabular}

Estes resultados indicam que as características individuais das árvores, tais como, grau de iluminação e forma da copa, são fatores que devem ser levados em consideração no monitoramento e execução das práticas de manejo florestal, pois interferem diretamente na produção da floresta remanescente.

\section{CONCLUSÕES}

- O sistema de manejo florestal adotado, utilizando técnicas de baixo impacto, mostrou-se eficiente para proporcionar a recuperação do povoamento florestal remanescente.

- 16 e 18 anos após a exploração, a floresta estudada apresentou estoques em área basal e volume do povoamento total e das espécies comerciais, estatisticamente iguais aos registrados antes da primeira exploração.

- As altas taxas de mortalidade observadas logo após a exploração, estão sendo compensadas pelo aumento do número de ingressos, tanto para o povoamento total, quanto para as espécies comerciais.

- Fatores ambientais como exposição a luz e a boa distribuição da copa, influenciam diretamente na velocidade do crescimento das árvores, por isso torna-se imprescindível considerar estes fatores para um melhor planejamento e execução das atividades de manejo florestal, visando a viabilidade do segundo ciclo de corte.

\section{REFERÊNCIAS}

CARVALHO, J. O. P. de; SILVA, J. N. M.; LOPES, J. do C. A. Growth rate of terra firme rain forest in brazilian amazon over an eight-year period in response to logging. Acta Amazônica, Manaus, v. 34, n. 2, p. 209-217, 2004.

CUNHA, T. A. Modelagem do Incremento de árvores individuais de Cedrela odorata L. na Floresta Amazônica. 87 f. Dissertação de Mestrado. Universidade Federal de Santa Maria -RS, 2009.

De GRAAF, N. R. de. A silvicultural system for natural regeneration of tropical rain forest in Suriname. Wageningen: Agricultural University, 1986, 250 p. 
FOOD AND AGRICULTURE ORGANIZATION (FAO). Global Forest Resources Assesment. Disponível em: <http://www.fao.org/docrep/013/i1757e/i1757e.pdf.> Acesso em: 25/09/2013.

FERREIRA, N. F. Análise da sustentabilidade do manejo florestal com base na avaliação de danos causados por exploração de impacto reduzido (eir) em floresta de terra firme no município de Paragominas-PA. $85 \mathrm{f}$. Dissertação de Mestrado. Universidade Federal Rural da Amazônia (UFRA), Belém, Pará, 2005.

FURTADO, C. S. Dinamica de uma floresta sob regime de manejo Sustentável em escala empresarial na Amazonia ocidental. Tese de Doutorado. Instituto Nacional de Pesquisas da Amazônia (INPA/UFAM), Manaus, Amazonas, 2009.

HIGUCHI, N.; SANTOS, J. dos; RIBEIRO, R. J.; FREITAS, J. V.; VIEIRA, G.; COIC, A. R.; MINETTE, L. J. Crescimento e incremento de uma floresta Amazônica de terra-firme manejada experimentalmente: Workshop Bionte - Aspectos Silviculturais e ecológicos do Manejo Florestal INPA. Manaus/AM,1997.

INSTITUTO BRASILEIRO DE GEOGRAFIA E ESTATÍSTICA (IBGE) - FUNDAÇÃO INSTITUTO BRASILEIRO DE GEOGRAFIA E ESTATÍSTICA. Manual técnico da vegetação brasileira: Série Manuais Técnicos de Geociências. Rio de Janeiro, 2012. 271 p.

KOTTEK, M. J.; GRIESER, C.; BECK, B.; RUDOLF, B.; RUBEL, F. World Map of Köppen-Geiger Climate Classification updated. Meteorol. Z., 15, 2006. p. 259-263.

LIMA, A. J. N. L. Avaliação de um sistema de Inventário Florestal Contínuo em áreas manejadas e não manejadas do Estado do Amazonas (AM). Tese de Doutorado, Instituto Nacional de Pesquisas da Amazônia, INPA/UFAM, Manaus, Amazonas, 2010.183 p.

LOPES, J. C. A. Demografia e flutuações temporais da regeneração natural após uma exploração florestal: FLONA do Tapajós- PA. Dissertação de Mestrado. Escola Superior de Agricultura Luiz de Queiroz. Universidade de São Paulo. 1993.

OLIVEIRA, L. C. Efeito da exploração da madeira e de diferentes intensidades de desbaste sobre a dinâmica da vegetação de uma área de 136 ha na Floresta Nacional do Tapajós. Tese de Doutorado, Escola Superior de Agricultura "Luiz de Queiroz"/USP, Piracicaba, São Paulo, 2005.

d'OLIVEIRA, M. V. N.; BRAZ, E. M. 2006. Estudo da dinâmica da floresta manejada no projeto de manejo florestal comunitário do PC Peixoto na Amazônia Ocidental. Acta Amazônica. 36(2): 177-182.

PMFS/Mil Madeiras. Plano de Manejo Florestal Sustentável da Mil Madeiras Preciosas/PWA. VI Reformulação. Itacoatiara/AM, 2013.

RADAMBRASIL. Programa de Integração Nacional. Levantamento de Recursos Naturais: Geologia, Geomorfologia, Pedologia, Vegetação e Uso Potencial da Terra. Santarém: DNPM, Ministério das Minas e Energia. v. 10. f SA21, 1978. 509 p.

SILVA, J. N. M.; de CARVALHO, J. O. P.; LOPES, J. C. A.; de OLIVEIRA, R. P., de OLIVEIRA, L. C. Growth and yield studies in the Tapajós region, Central Brazilian Amazon. Commonwealth Forestry Rev. 75 (4), 325-329. 1996.

SILVA, E. J. V. Dinâmica de florestas manejadas e sob exploração convencional na Amazônia Oriental. Tese de Doutorado. Escola de Engenharia de São Carlos. Universidade de São Paulo. 2004.

SILVA, J. N. M.; LOPES, J. C. A.; OLIVEIRA, L. C.; SILVA, S. M. A.; CARVALHO, J. O. P.; COSTA, D. H. M.; MELO, M. S.; TAVARES, M. J. M. Diretrizes para a instalação e medição de parcelas permanentes em florestas naturais da Amazônia Brasileira. Embrapa Amazônia Oriental, Belém, Pará. 2005.

SOUZA, C. R. Dinâmica de carbono em floresta explorada e em floresta nativa não explorada na Amazônia-Manaus. Tese de Doutorado. Instituto Nacional de Pesquisas da Amazônia (INPA/UFAM), Manaus, Amazonas. 2012.

TEIXEIRA, L. M.; JEFFREY, Q.; CHAMBERS, J. Q.; SILVA, A. R. E.; LIMA, A. J. N.; CARNEIRO, V. M. C.; SANTOS, J. dos; HIGUCHI, N. Projeção da dinâmica da floresta natural de Terra-firme, região de Manaus - AM, com o uso da cadeia de transição probabilística de Markov. Acta Amazonica, Manaus, v.37, n. 3, p. 377-384, 2007.

TOLEDO, J. J.; MAGNUSSON, W. E.; CASTILHO, C. V.; NASCIMENTO, H. E. M. 2011. How much variation in tree mortality is predicted by soil and topography in Central Amazonia? Forest Ecology and Management, 262: 331-338.

FLORESTA, Curitiba, PR, v. 47, n. 1, p. 55 - 63, jan. / mar. 2017.

Souza, M. A. S. de et al.

ISSN eletrônico 1982-4688

DOI: $10.5380 /$ rf.v47i1.43312 